\title{
Quadratic Spline and Two-Point Boundary Value Problem
}

\author{
By \\ Manabu SAKAI* and Riaz A. USMANI**
}

\section{$\S 1$. Introduction}

We shall concern ourself with the solution of real, nonlinear two-point boundary value problems of the form

$$
\begin{aligned}
& x^{\prime \prime}(t)=f\left(t, x(t), x^{\prime}(t)\right), t \in[0,1] \\
& a_{0} x(0)-b_{0} x^{\prime}(0)=c_{0} \\
& a_{1} x(1)+b_{1} x^{\prime}(1)=c_{1}
\end{aligned}
$$

where $f(t, x, y)$ is defined and twice continuously differentiable in a region $D$ of the $(t, x, y)$-space intercepted by two hyperplanes $t=0$ and $t=1$. The analytical solution of (1.1) for arbitrary choices of the function $f$ cannot be found in general. We usually resort to some numerical method for obtaining an approximate solution of the problem (1.1). The standard numerical methods for the numerical treatment of (1.1) consist of finite difference methods, shooting methods, RayleighRitz and Galerkin's procedure and collocation methods. A long list of references of all of these methods is given by Keller [4] in [2]. The subject of obtaining spline solutions for the initial as well as boundary value problems is briefly discussed in [1]. Since then many papers have appeared dealing with the continuous approximation of $x(t)$ satisfying (1.1) via cubic and quintic splines mainly (see $[3,9]$ ). The collocation methods using spline functions have been developed and analysed by Sakai (see [5], [6], [7]) again employing cubic and quintic splines at equi-distant knots. Recently Usmani and Sakai [11] have also used quadratic spline function for solving a two-point boundary value problem involving a fourth order differential equation.

In this brief report, we propose a second order collocation method using quadratic spline employing $B$-splines. In the sequel, it will be shown that our method is an $O\left(h^{2}\right)$-convergent procedure. In the last section some numerical evidence is included to show the practical applicability of our method by solving

Communicated by S. Hitotumatu, May 28, 1981.

* Department of Mathematics, Faculty of Science, Kagoshima University, Kagoshima, Japan, 890

** On leave from the Department of Applied Mathematics, University of Manitoba, Winnipeg, Canada, R3T 2N2. 
nonlinear as well as linear boundary value problems of the form (1.1). We begin the next section by giving a formal definition of our numerical method.

\section{$\S 2$. Definition of Our Method}

We first rewrite the differential system into an equivalent form

$$
\begin{aligned}
& x^{\prime}(t)=z(t) \\
& z^{\prime}(t)=f(t, x(t), z(t)) \\
& a_{0} x(0)-b_{0} z(0)=c_{0}, \quad a_{1} x(1)+b_{1} z(1)=c_{1} .
\end{aligned}
$$

Now making use of $B$-spline $Q_{m+1}(t)=(1 / m !) \sum_{i=1}^{m}(-1)^{i}\left(\begin{array}{c}m+1 \\ i\end{array}\right)(t-i)_{+}^{m}$, we consider quadratic and piecewies linear functions of the form

$$
x_{h}(t)=\sum_{i=-2}^{n-1} \alpha_{i} Q_{3}(t / h-i), \quad z_{h}(t)=\sum_{i=-1}^{n-1} \beta_{i} Q_{2}(t / h-i) \quad(n h=1)
$$

with undetermined coefficients $\left(\alpha_{-2}, \alpha_{-1}, \alpha_{0}, \cdots, \alpha_{n-1}\right)$ and $\left(\beta_{-1}, \beta_{0}, \cdots, \beta_{n-1}\right)$. The above $x_{h}(t)$ and $z_{h}(t)$ will be approximate solutions to (2.1) if they satisfy

$$
\begin{aligned}
& x_{h}^{\prime}(t)=z_{h}(t) \\
& z_{h}^{\prime}(t)=P f\left(t, x_{h}(t), z_{h}(t)\right) \\
& a_{0} x_{h}(0)-b_{0} z_{h}(0)=c_{0}, \quad a_{1} x_{h}(1)+b_{1} z_{h}(1)=c_{1} .
\end{aligned}
$$

Here $P$ is an operator such that

$$
(P g)(t)=\sum_{i=0}^{n-1} g\left(t_{i+1 / 2}\right) \chi(t / h-i), \quad t_{i+1 / 2}=\left(t_{i+1}+t_{i}\right) / 2
$$

where $\chi(t)$ is a step function with the property

$$
\chi(t)= \begin{cases}1, & 0<t<1 \\ 0, & \text { otherwise. }\end{cases}
$$

From (2.3), we have the following system of nonlinear equations connecting $\alpha_{i}$ and $\beta_{i}$ :

$$
\left\{\begin{array}{l}
F_{-1}(\alpha, \beta)=\left(a_{0} / 2\right)\left(\alpha_{-1}+\alpha_{-2}\right)-b_{0} \beta_{-1}-c_{0}=0 ; \\
F_{i}(\alpha, \beta)=(1 / h)\left(\alpha_{i-1}-\alpha_{i-2}\right)-\beta_{i-1}=0, \quad i=0(1) n ; \\
F_{n+i}(\alpha, \beta)=(1 / h)\left(\beta_{i-1}-\beta_{i-2}\right)-f\left(t_{i-1 / 2},(1 / 8)\left(\alpha_{i-1}\right.\right. \\
\left.\left.\quad+6 \alpha_{i-2}+\alpha_{i-3}\right),(1 / 2)\left(\beta_{i-1}+\beta_{i-2}\right)\right)=0, \quad i=1(1) n ; \\
F_{2 n+1}(\alpha, \beta)=\left(a_{1} / 2\right)\left(\alpha_{n-1}+\alpha_{n-2}\right)+b_{1} \beta_{n-1}-c_{1}=0 .
\end{array}\right.
$$

In any practical computations, however, we may use the following system of nonlinear equations containing $\alpha_{i}$ alone:

$$
\left\{\begin{array}{c}
\left(a_{0} / 2\right)\left(\alpha_{-1}+\alpha_{-2}\right)-\left(b_{0} / h\right)\left(\alpha_{-1}-\alpha_{-2}\right)=c_{0}, \\
\left(1 / h^{2}\right)\left(\alpha_{i-1}-2 \alpha_{i-2}+\alpha_{i-3}\right)=f\left(t_{i-1 / 2},(1 / 8)\left(\alpha_{i-1}\right.\right. \\
\left.\left.\quad+6 \alpha_{i-2}+\alpha_{i-3}\right),(1 / 2 h)\left(\alpha_{i-1}-\alpha_{i-3}\right)\right), \quad i=1(1) n, \\
\left(a_{1} / 2\right)\left(\alpha_{n-1}+\alpha_{n-2}\right)+\left(b_{1} / h\right)\left(\alpha_{n-1}-\alpha_{n-2}\right)=c_{1} .
\end{array}\right.
$$


The system (2.6) is easily obtained from (2.5) on eliminating $\beta_{i}$.

Our object now is to show that under certain conditions the system of nonlinear equations (2.5) has a unique solution. To this end, we assume that the system (2.1) has an isolated solution $(\hat{x}(t), \hat{z}(t))$ satisfying the internality condition

$$
U=\{(t, x, z):|x-\hat{x}(t)|+|z-\hat{z}(t)| \leqq \delta, \quad t \in[0,1]\} \subset D
$$

for some $\delta>0$. A solution $(\hat{x}(t), \hat{z}(t))$ is isolated if and only if

$$
G=\left[\begin{array}{ll}
a_{0} & -b_{0} \\
a_{1} \phi_{0}(1)+b_{1} \phi_{0}^{\prime}(1) & a_{1} \phi_{1}(1)+b_{1} \phi_{1}^{\prime}(1)
\end{array}\right]
$$

is nonsingular, where $\phi_{0}$ and $\phi_{1}$ are the solutions of the initial value problems

$$
\left\{\begin{array}{l}
\phi_{j}^{\prime \prime}=f_{2}(t, \hat{x}, \hat{z}) \phi_{j}+f_{3}(t, \hat{x}, \hat{z}) \phi_{j}^{\prime} \\
\phi_{j}^{(i)}(0)=\delta_{i j}, \quad(i, j=0,1), \quad\left(f_{i}\left(x_{1}, x_{2}, x_{3}\right)=\frac{\partial f}{\partial x_{i}}, i=2,3\right) .
\end{array}\right.
$$

Corresponding to $(\hat{x}(t), \hat{z}(t))$, we can determine quadratic spline and piecewise linear functions

$$
\hat{x}_{h}(t)=\sum \hat{\alpha}_{i} Q_{3}(t / h-i), \quad \hat{z}_{h}(t)=\hat{x}_{h}^{\prime}(t)=\sum \hat{\beta}_{i} Q_{2}(t / h-i)
$$

so that

$$
\begin{cases}\hat{x}_{h}\left(t_{i+1 / 2}\right)=\hat{x}\left(t_{i+1 / 2}\right), & i=0(1) n-1 \\ \hat{x}_{n}^{\prime \prime}\left(t_{i+1 / 2}\right)=\hat{x}^{\prime \prime}\left(t_{i+1 / 2}\right), & i=0, n-1 .\end{cases}
$$

By the use of consistency relations

$$
\left\{\begin{aligned}
\left(1 / h^{2}\right) & \left\{\hat{x}_{h}\left(t_{i+3 / 2}\right)-2 \hat{x}_{h}\left(t_{i+1 / 2}\right)+\hat{x}_{h}\left(t_{i-1 / 2}\right)\right\} \\
& =(1 / 8)\left\{\hat{x}_{h}^{\prime \prime}\left(t_{i+3 / 2}\right)+6 \hat{x}_{h}^{\prime \prime}\left(t_{i+1 / 2}\right)+\hat{x}_{h}^{\prime \prime}\left(t_{i-1 / 2}\right)\right\}, \\
(1 / 2 h) & \left\{\hat{x}_{h}\left(t_{i+3 / 2}\right)-\hat{x}_{h}\left(t_{i-1 / 2}\right)\right\}=(1 / 8)\left\{\hat{x}_{h}^{\prime}\left(t_{i+3 / 2}\right)+6 \hat{x}_{h}^{\prime}\left(t_{i+1 / 2}\right)+\hat{x}_{h}^{\prime}\left(t_{i-1 / 2}\right)\right\}, \\
\hat{x}_{h}^{\prime}\left(t_{1 / 2}\right) & =(1 / h)\left\{\hat{x}_{h}\left(t_{3 / 2}\right)-\hat{x}_{h}\left(t_{1 / 2}\right)\right\}-(h / 8)\left\{\hat{x}_{h}^{\prime \prime}\left(t_{3 / 2}\right)+3 \hat{x}_{h}^{\prime \prime}\left(t_{1 / 2}\right)\right\},
\end{aligned}\right.
$$

we can easily deduce

$$
\begin{aligned}
& \hat{x}_{h}^{\prime \prime}\left(t_{i+1 / 2}\right)=\hat{x}^{\prime \prime}\left(t_{i+1 / 2}\right)+O\left(h^{2}\right), \quad i=0(1) n-1 \\
& \left\|\hat{x}_{h}^{(m)}-\hat{x}^{(m)}\right\|=\max \left|\hat{x}_{h}^{(m)}(t)-\hat{x}^{(m)}(t)\right|=O\left(h^{3-m}\right) \quad(m=0,1),
\end{aligned}
$$

(see the details of (2.12) and (2.13) in [11]).

From the preceding estimates (2.13), it easily follows that

$$
\|F(\hat{\alpha}, \hat{\beta})\|=O\left(h^{2}\right),
$$

where for any finite dimensional vector, we shall denote its maximum norm by $\|\cdot\|$.

\section{$\S 3$. Existence and Convergence of Spline Approximations}

Let $J(\alpha, \beta)$ be the Jacobian matrix of $F(\alpha, \beta)$ with respect to $(\alpha, \beta)$. In order to investigate the properties of $J(\hat{\alpha}, \hat{\beta})$, let us consider a linear system 


$$
\begin{gathered}
J(\hat{\alpha}, \hat{\beta})(\xi, \eta)=(\lambda, \mu) \\
\xi=\left(\xi_{-2}, \xi_{-1}, \cdots, \xi_{n-1}\right), \quad \eta=\left(\eta-1, \eta_{0}, \cdots, \eta_{n-1}\right) \\
\lambda=\left(\lambda_{-1}, \lambda_{0}, \cdots, \lambda_{n}\right), \quad \mu=\left(\mu_{1}, \mu_{2}, \cdots, \mu_{n+1}\right) .
\end{gathered}
$$

where

Corresponding to $\xi$ and $\eta$, we consider quadratic spline and piecewise linear functions $\phi(t)$ and $\phi(t)$

$$
\phi(t)=\sum_{i=-2}^{n-1} \xi_{i} Q_{3}(t / h-i) \text { and } \phi(t)=\sum_{i=-1}^{n-1} \eta_{i} Q_{2}(t / h-i) .
$$

From (3.1), we have

$$
\left\{\begin{array}{l}
a_{0} \phi(0)-b_{0} \phi(0)=\lambda_{-1} \\
\phi^{\prime}\left(t_{i}\right)-\phi\left(t_{i}\right)=\lambda_{i}, \quad(i=0(1) n) \\
\phi^{\prime}\left(t_{i-1 / 2}\right)=f_{2}\left(t_{i-1 / 2}, \hat{x}_{h}\left(t_{i-1 / 2}\right), \hat{z}_{h}\left(t_{i-1 / 2}\right)\right) \phi\left(t_{i-1 / 2}\right) \\
\quad+f_{3}\left(t_{i-1 / 2}, \hat{x}_{h}\left(t_{i-1 / 2}\right), \hat{z}_{h}\left(t_{i-1 / 2}\right)\right) \psi\left(t_{i-1 / 2}\right)+\mu_{i} \quad(i=1(1) n), \\
a_{1} \phi(1)+b_{1} \phi(1)=\mu_{n+1} .
\end{array}\right.
$$

Let $\lambda(t)$ and $\mu(t)$ be piecewise linear and step functions satisfying the conditions:

$$
\lambda\left(t_{i}\right)=\lambda_{i}, \quad(i=0(1) n) \text { and } \mu\left(t_{i-1 / 2}\right)=\mu_{i} \quad(i=1(1) n),
$$

then we have

$$
\left\{\begin{array}{c}
\phi^{\prime}(t)=\psi(t)+\lambda(t), \quad 0 \leqq t \leqq 1 \\
\phi^{\prime}(t)=P\left[f_{2}\left(t, \hat{x}_{h}(t), \hat{z}_{h}(t)\right) \phi(t)+f_{3}\left(t, \hat{x}_{h}(t), \hat{z}_{h}(t)\right) \psi(t)\right]+\mu(t), \\
t_{i}<t<t_{i+1}, \quad i=0(1) n-1 \\
a_{0} \phi(0)-b_{r} \phi(0)=\lambda_{-1}, \quad a_{1} \phi(1)+b_{1} \phi(1)=\mu_{n+1},
\end{array}\right.
$$

from which follows

$$
\left\{\begin{array}{l}
\phi^{\prime}(t)-\phi(t)=\lambda(t) \\
\phi^{\prime}(t)-f_{2}(t, \hat{x}(t), \hat{z}(t)) \phi(t)-f_{3}(t, \hat{x}(t), \hat{z}(t)) \phi(t)=R+\mu(t), \quad t \neq t_{i} \\
a_{0} \phi(0)-b_{0} \phi(0)=\lambda_{-1}, \quad a_{1} \phi(1)+b_{1} \phi(1)=\mu_{n+1},
\end{array}\right.
$$

where $R=-(I-P)\left[f_{2} \phi+f_{3} \phi\right]+O(h)[\|\phi\|+\|\phi\|]$ (I the unit operator) by the use of (2.13).

Since $G$ is nonsingular due to the assumption that (2.1) has an isolated solution $(\hat{x}, \hat{z})$, there exists the Green function $H(t, s)$ such that

$$
H(t, s)= \begin{cases}\Phi(t)\left[E-G^{-1} M_{0} \Phi(1)\right] \Phi^{-1}(s), & 0 \leqq s \leqq t \\ -\Phi(t) G^{-1} M_{0} \Phi(1) \Phi^{-1}(s), & s<t \leqq 1,\end{cases}
$$

where

$$
M_{0}=\left[\begin{array}{cc}
0 & 0 \\
a_{1} & b_{1}
\end{array}\right], \quad E=\left[\begin{array}{ll}
1 & 0 \\
0 & 1
\end{array}\right], \quad \Phi(t)=\left[\begin{array}{ll}
\phi_{0} & \phi_{1} \\
\phi_{0}^{\prime} & \phi_{1}^{\prime}
\end{array}\right] .
$$

By making use of the Green function, we obtain

$$
\left[\begin{array}{l}
\phi(t) \\
\phi(t)
\end{array}\right]=\Phi(t) G^{-1}\left[\begin{array}{l}
\lambda_{-1} \\
\mu_{n+1}
\end{array}\right]+\int_{0}^{1} H(t, s)\left[\begin{array}{l}
\lambda(s) \\
R+\mu(s)
\end{array}\right] d s .
$$


From now on, for any finite dimensional vector $v=\left(v_{i}\right)$, we denote $\max _{\boldsymbol{i}}\left|v_{i}\right|$ by $\|v\|$, and a generic constant independent of $h$ by $C$. Since

we have

$$
\begin{aligned}
\|R\|_{\left[t_{i}, t_{i+1}\right]} & \leqq C h\left(\|\phi\|+\left\|\phi^{\prime}\right\|+\|\phi\|+\left\|\phi^{\prime}\right\|_{\left[t_{i}, t_{i+1}\right]}\right) \\
& \leqq C h\left(\|\phi\|+\|\phi\|+\|\mu\|_{\left[t_{i}, t_{i+1}\right]}+\|\lambda\|\right) \quad \text { ([5]), }
\end{aligned}
$$

$$
\|R\|_{\left[t_{i}, t_{i+1}\right]} \leqq C h\left(\|\phi\|+\|\phi\|+\|\mu\|_{\left[t_{i}, t_{i+1}\right]}+\|\lambda\|\right) .
$$

On applying inequality (3.8) to (3.7) we have

$$
\|\phi\|,\|\phi\| \leqq C\|(\lambda, \mu)\| \quad \text { for any } h<h_{0}
$$

provided that $h_{0}$ is sufficiently small. Since $\|\phi\| \geqq C\|\xi\|$, $\|\phi\| \geqq C\|\eta\|$, we have

$$
\|(\xi, \eta)\| \leqq C\|(\lambda, \mu)\| \quad \text { for any } h<h_{0} \text {. }
$$

From (3.1) and (3.9) it follows that the martix $J(\hat{\alpha}, \hat{\beta})$ is nonsingular and in addition

$$
\left\|J^{-1}(\hat{\alpha}, \hat{\beta})\right\| \leqq C \quad \text { for } h<h_{0} .
$$

Let $\Omega_{h_{0}}=\left\{(\alpha, \beta):\|\alpha-\hat{\alpha}\|+\|\beta-\hat{\beta}\| \leqq \delta_{0}=\delta-\left\|\hat{x}-\hat{x}_{h}\right\|-\left\|\hat{z}-\hat{z}_{h}\right\|\right\}$ for $h<h_{0}$ provided $h_{0}$ is sufficiently small, then we have

$$
\left\|J\left(\alpha_{1}, \beta_{1}\right)-J\left(\alpha_{2}, \beta_{2}\right)\right\| \leqq C\left\|\left(\alpha_{1}, \beta_{1}\right)-\left(\alpha_{2}, \beta_{2}\right)\right\|
$$

for $\left(\alpha_{1}, \beta_{1}\right)$ and $\left(\alpha_{2}, \beta_{2}\right) \in \Omega_{h_{0}}$.

The inequalities (2.14), (3.10) and (3.11) satisfy all the conditions of NewtonKantorovitoch's theorem (see Rall [8]). Thus the system of nonlinear equations $F(\alpha, \beta)=0$ has one and only one solution $(\bar{\alpha}, \bar{\beta})$ in the neigbourhood of $(\hat{\alpha}, \hat{\beta})$ so that

$$
\|(\bar{\alpha}, \bar{\beta})-(\hat{\alpha}, \hat{\beta})\|=O\left(h^{2}\right)
$$

Hence we have

Theorem. In a sufficiently small neigbourhood of the isolated solution $\hat{x}(t)$, there exists an approximate solution

$$
\bar{x}_{h}(t)=\sum \bar{\alpha}_{i} Q_{3}(t / h-i)
$$

so that

$$
\left\|\hat{x}^{(m)}-\bar{x}_{h}^{(m)}\right\|=O\left(h^{2}\right), \quad(m=0,1), \quad(h \rightarrow 0) .
$$

\section{§4. Numerical Illustrations}

We now illustrate the usefulness of the method of this paper by applying it to the following two-point boundary value problems of the form (1.1).

Example 1. $x^{\prime \prime}=100 x, x(0)=x(1)=1$ with the solution

$$
\hat{x}(t)=\cosh (10 t-5) / \cosh 5 \text {. }
$$


Example 2. $x^{\prime \prime}=\left(2 / t^{2}\right) x-1 / t, \quad x(2)=x(3)=0, \quad$ with the solution $\hat{x}(t)=$ $\left(19 t-5 t^{2}-36 / t\right) / 38$.

Example 3. $x^{\prime \prime}=\left[e^{2 x}+\left(x^{\prime}\right)^{2}\right] / 2, x(0)-x^{\prime}(0)=1, x(1)+x^{\prime}(1)=-0.5-\ln 2$, with the exact solution $\hat{x}(t)=-1 n(1+t)$.

Example 4. $x^{\prime \prime}=\left(x+t x^{\prime}\right) /(1+t), x(0)-2 x^{\prime}(0)=-1, x(1)+2 x^{\prime}(1)=3 e$, with the exact solution $\hat{x}(t)=e^{t}$.

All the computations were performed in double precision arithmetic in order to keep the rounding errors to a minimum.

We solved all the four examples with different step-sizes $h=2^{-m}, m=1,2$, $\cdots, 5$. In Table 1 , we display the computed solution of Example 1 with $h=1 / 32$ for $0 \leqq t \leqq 1 / 2$, for the solution of this problem is symetrical about the line $t=1 / 2$. In Table 2, we list the observed maximum errors for $0 \leqq t \leqq 1$. The entries of Table 2 do confirm that our numerical procedure is an $O\left(h^{2}\right)$-convergent process. For example the observed maximum errors for Example 4 with $h=1 / 16$ and $h=1 / 32$ are $0.25359 \times 10^{-3}$, and $0.63404 \times 10^{-4}$ respectively. Thus, on reducing the step-size from $h=1 / 16$ to $1 / 32$, the maximum absolute error is reduced approximately by a factor of $1 / 4$.

We remark that by using Richardson's extrapolation technique the accuracy of our computed solution can be improved at mesh points. The extrapolated solution $\left\{\left[4 \bar{x}_{h / 2}\left(t_{i}\right)-\bar{x}_{h}\left(t_{i}\right)\right] / 3, i=0(1) n\right\}$ will in fact be $O\left(h^{4}\right)$-convergent.

Table 1 (Example 1: $\left.e_{i}=\left|\hat{x}\left(t_{i}\right)-\bar{x}_{h}\left(t_{i}\right)\right| \times 10^{3}, h=1 / 32\right)$

\begin{tabular}{rrrrrrrrr}
\hline$t_{i}$ & $1 / 16$ & $2 / 16$ & $3 / 16$ & $4 / 16$ & $5 / 16$ & $6 / 16$ & $7 / 16$ & $8 / 16$ \\
\hline$e_{i}$ & 0.471 & 0.740 & 0.596 & 0.430 & 0.297 & 0.205 & 0.155 & 0.138 \\
\hline
\end{tabular}

Table 2

Observed max. errors

\begin{tabular}{rcccc}
\hline \multicolumn{1}{c}{$h$} & Example 1 & Example 2 & Example 3 & Example 4 \\
\hline $1 / 2$ & $0.218-0$ & $0.297-3$ & $0.660-2$ & $0.161-1$ \\
$1 / 4$ & $0.499-1$ & $0.793-4$ & $0.171-2$ & $0.405-2$ \\
$1 / 8$ & $0.140-1$ & $0.206-4$ & $0.432-3$ & $0.101-2$ \\
$1 / 16$ & $0.306-2$ & $0.520-5$ & $0.108-3$ & $0.254-3$ \\
$1 / 32$ & $0.758-3$ & $0.130-5$ & $0.271-4$ & $0.634-4$ \\
\hline
\end{tabular}


Table 3

Observed errors based on $h^{2}$-extrapolation at $t=1 / 2$.

\begin{tabular}{ccccc}
\hline \multicolumn{1}{c}{$h$} & Example 1 & Example 2 & Example 3 & Example 4 \\
\hline $1 / 2$ & $0.677-1$ & $0.670-5$ & $0.299-4$ & $0.121-3$ \\
$1 / 4$ & $0.522-3$ & $0.456-6$ & $0.196-5$ & $0.760-5$ \\
$1 / 8$ & $0.607-4$ & $0.299-7$ & $0.124-6$ & $0.477-6$ \\
$1 / 16$ & $0.413-5$ & $0.188-8$ & $0.777-8$ & $0.298-7$ \\
$1 / 32$ & & & & \\
\hline
\end{tabular}

\section{References}

[1] Ahlberg, J., Nilson, E. and Walsh, J., The theory of splines and their applications, Academic Press, New York, 1967.

[2] Aziz, A.K. (Editor), Numerical solutions of boundary value problems for ordinary differential equations, Academic Press, New York, 1975.

[3] De Boor, C., A practical guide to splines, Springer-Verlag, New York, 1978.

[4] Keller, H.B., Numerical solution of boundary value problems for ordinary differential equations: Survey and some recent results on difference methods, in [2], 27-88.

[5] Sakai, M., Piecewise cubic spline interpolation and two point boundary value problems, Publ. RIMS, Kyoto Univ., 7 (1971/1972), 345-362.

[6] - Spline interpolation and two-sided approximate methods for two-point boundary value problems, Reports of the Faculty of Science, Kagoshima Univ., 11 (1978), 1-19.

[7] - Two-sided spline approximate methods for the two point boundary value problems, Reports of the Faculty of Science, Kagoshima Univ., 13 (1980), 15-31.

[8] Rall, L.B., Computational solutions of nonlinear operator equations, John Wiley \& Sons, New York, 1969.

[9] Schultz, M., Spline analysis, Prentice-Hall, Englewood Cliffs, N. J., 1973.

[10] Stepleman, R., Tridiagonal fourth order approximations to general two-point nonlinear boundary value problems with mixed boundary conditions, Mathematics of Computation, 30 (1976), 92-103.

[11] Usmani, R. and Sakai, M., A note on the quadratic spline interpolation at midpoints, 1982, to appear in BIT.

[12] — - On the use of a two-point boundary value problem involving a fourth order differential equation, 1981 (unpublished). 
